\title{
Clinical implication of impaired skin microvascular function in patients with hypertension
}

\author{
Tatsuya Maruhashi ${ }^{1}$ Yukihito Higashi ${ }^{1,2}$
}

Received: 29 November 2021 / Accepted: 3 December 2021 / Published online: 27 December 2021

(c) The Japanese Society of Hypertension 2021

Several noninvasive methods for imaging microcirculation have been developed and used in clinical practice. Laser Doppler perfusion imaging (LDPI) is a laser-based optical imaging technique that has occasionally been used for the assessment of skin microcirculation. A major limitation of the standard LDPI technique is its low temporal resolution; a measurement time of one minute is required for scanning. Therefore, the standard LDPI technique is not suitable for skin microvascular function tests with a dynamic process [1]. The development of laser Doppler flowmetry (LDF) using the Doppler shift of laser light as the information carrier has enabled continuous measurement of tissue blood flow, making it possible to evaluate skin microvascular function $[2,3]$. However, the spatial resolution and reproducibility of LDF are poor [4]. An alternative laser-based optical imaging method for microcirculation is laser speckle contrast imaging (LSCI), which has an excellent correlation with LDPI [5]. This method relies on the speckle phenomenon to create dynamic two-dimensional maps of microvascular perfusion and visualizes blood flux in real time with high spatial and temporal resolution. Therefore, the LSCI technique enables dynamic mapping of skin blood flux in almost real time with higher reproducibility than that of LDF, making the LSCI technique a fascinating tool for the assessment of skin microvascular function [6].

Skin microcirculation has been regarded as a potentially representative vascular bed for the assessment of microvascular function and understanding of the pathophysiological associations between microvascular dysfunction and

Tatsuya Maruhashi

maru0512@hiroshima-u.ac.jp

1 Department of Cardiovascular Regeneration and Medicine, Research Institute for Radiation Biology and Medicine, Hiroshima University, Hiroshima, Japan

2 Division of Regeneration and Medicine, Medical Center for Translational and Clinical Research, Hiroshima University Hospital, Hiroshima, Japan disease states [6]. There are two major types of skin microvascular function tests: tests with pharmacological interventions using acetylcholine, bradykinin, histamine, methacholine, phenylephrine, or sodium nitroprusside and tests with nonpharmacological interventions, including local cooling tests, local thermal hyperemia tests, pressureinduced vasodilation tests, transient hyperemic response tests, and postocclusive reactive hyperemia (PORH) tests [6]. Skin microvascular function tests have been performed in a variety of disease states, including type 2 diabetes, dyslipidemia, renal disease, peripheral artery disease, coronary artery disease, heart failure, Alzheimer's disease, and systemic sclerosis [6]. However, there is little information on skin microvascular function evaluated by the LSCI technique in patients with hypertension.

In the current issue of Hypertension Research, Antonios et al. evaluated skin microvascular function by using the LSCI technique coupled with PORH in untreated patients with essential hypertension, patients with masked hypertension, and control subjects without hypertension [7]. Skin microcirculation was measured at two skin sites $>10 \mathrm{~mm}^{2}$ that were randomly chosen on the ventral surface of the forearm. PORH was induced by inflating a pneumatic cuff wrapped around the upper arm at $250 \mathrm{~mm} \mathrm{Hg}$ for $5 \mathrm{~min}$. Skin microvascular function was defined as a percentage increase in flux from baseline to the peak postocclusive response (base to peak flux, \%) or the amplitude of the PORH response (PORH amplitude) expressed as the peak cutaneous vascular conductance (CVC) minus baseline CVC. CVC was calculated as the ratio of mean flux in each respective period divided by mean blood pressure. They demonstrated that both base-to-peak flux and PORH amplitude were significantly impaired in patients with essential hypertension and patients with masked hypertension compared to those in subjects without hypertension, that both base-to-peak flux and PORH amplitude were negatively correlated with office blood pressure and ambulatory blood pressure and that hypertension was significantly associated with lower base-to-peak flux and 
PORH amplitude after adjusting for other confounding factors, indicating that hypertension is associated with impaired skin microvascular function. In multivariate analysis, not only hypertension but also total cholesterol and smoking were significantly associated with impaired skin microvascular function. These findings indicate the possibility that skin microvascular function assessed by the LSCI technique coupled with PORH can be used as a vascular biomarker.

An ideal vascular function test as a vascular biomarker should reflect and follow the disease severity and, thus, should be reversible with interventions. In addition, an ideal vascular function test should serve as a prognostic marker; improvement of vascular function should be accompanied by a reduction in the risk of cardiovascular events. Several noninvasive vascular function tests, including flowmediated vasodilation and reactive hyperemia peripheral arterial tonometry as indices of endothelial function, nitroglycerine-induced vasodilation as an index of vascular smooth muscle function, and brachial-ankle pulse wave velocity and cardio-ankle vascular index as indices of arterial stiffness, have been developed and used in clinical practice $[8,9]$. In clinical settings, the results of vascular function tests are expected to serve as vascular biomarkers that provide additive information for cardiovascular risk stratification and treatment optimization. A skin microvascular function test using the LSCI technique coupled with PORH is a relatively operator-independent and simple method with high reproducibility. Therefore, this vascular function test has the potential to be used as a vascular biomarker in clinical practice. The results of the present study showed that skin microvascular function was negatively correlated with blood pressure, suggesting that skin microvascular function reflects disease severity in patients with untreated hypertension. However, it is unknown whether blood pressure-lowering therapy, including nonpharmacological interventions through lifestyle modifications such as dietary sodium restriction, aerobic exercise, and body weight reduction and pharmacological interventions with antihypertensive drugs, improves skin microvascular function in patients with hypertension. It is also unknown whether skin microvascular function serves as a prognostic marker of cardiovascular events. Further studies are needed to determine whether skin microvascular function tests using the LSCI technique coupled with PORH can serve as a vascular biomarker that provides additive information for cardiovascular risk stratification beyond blood pressure in patients with hypertension. For clinical use, it is also necessary to standardize the measurement method and establish diagnostic criteria for skin microvascular dysfunction for appropriate interpretation of the test results.

Coronary microvascular dysfunction has attracted much attention as a potential mechanism of myocardial ischemia since patients with coronary microvascular dysfunction are at increased risk of cardiovascular events. Coronary microvascular function is often evaluated by coronary flow reserve (CFR), which is calculated as the ratio of coronary blood flow during maximal vasodilation to baseline coronary blood flow in response to vasodilator stimuli. However, this method is limited by its invasive nature, and thus, repeated measurements of CFR are clinically difficult to perform. Khan et al. reported that there was a positive correlation between coronary velocity reserve measured by transthoracic echocardiography during intravenous adenosine infusion and skin microvascular function measured by laser Doppler imaging during iontophoresis of acetylcholine and sodium nitroprusside, suggesting that skin microvascular function reflects coronary microvascular function [10]. If there is a correlation between CFR and skin microvascular function assessed by the LSCI technique coupled with PORH, the skin microvascular function test using the LSCI technique coupled with PORH can potentially provide useful information not only for cardiovascular risk stratification but also for the diagnosis of coronary microvascular dysfunction.

In summary, this study has shown a significant association between hypertension and impaired skin microvascular function assessed by the LSCI technique coupled with PORH. The most important purpose of a vascular function test is to assess cardiovascular risk and to optimize interventions for preventing future cardiovascular events. Future studies investigating the role of a skin microvascular function test as a vascular biomarker may further enhance the clinical implications of this vascular function test in patients with cardiovascular risk factors.

\section{Compliance with ethical standards}

Conflict of interest The authors declare no competing interests.

Publisher's note Springer Nature remains neutral with regard to jurisdictional claims in published maps and institutional affiliations.

\section{References}

1. Eriksson S, Nilsson J, Sturesson C. Non-invasive imaging of microcirculation: a technology review. Med Devices. 2014;7:445-52.

2. Seliger SL, Salimi S, Pierre V, Giffuni J, Katzel L, Parsa A. Microvascular endothelial dysfunction is associated with albuminuria and CKD in older adults. BMC Nephrol. 2016;17:82.

3. Yamamoto-Suganuma R, Aso Y. Relationship between postocclusive forearm skin reactive hyperaemia and vascular disease in patients with Type 2 diabetes-a novel index for detecting micro- and macrovascular dysfunction using laser Doppler flowmetry. Diabet Med. 2009;26:83-8.

4. Roustit M, Blaise S, Millet C, Cracowski JL. Reproducibility and methodological issues of skin post-occlusive and thermal hyperemia assessed by single-point laser Doppler flowmetry. Microvascular Res. 2010;79:102-8. 
5. Millet C, Roustit M, Blaise S, Cracowski JL. Comparison between laser speckle contrast imaging and laser Doppler imaging to assess skin blood flow in humans. Microvascular Res. 2011;82:147-51.

6. Mahe G, Humeau-Heurtier A, Durand S, Leftheriotis G, Abraham P. Assessment of skin microvascular function and dysfunction with laser speckle contrast imaging. Circ Cardiovasc Imaging. 2012;5:155-63.

7. Antonios L, Areti T, Konstantina D, Panagiotis D, Nikolaos K, Panagiota A, et al. Skin microvascular function as assessed with laser speckle contrast imaging is impaired in untreated essential and masked hypertensives. Hypertens Res. 2021 (in press).
8. Tanaka A, Tomiyama H, Maruhashi T, Matsuzawa Y, Miyoshi T, Kabutoya T, et al. Physiological diagnostic criteria for vascular failure. Hypertension 2018;72:1060-71.

9. Maruhashi T, Kihara Y, Higashi Y. Assessment of endotheliumindependent vasodilation: from methodology to clinical perspectives. J Hypertension. 2018;36:1460-7.

10. Khan F, Patterson D, Belch JJ, Hirata K, Lang CC. Relationship between peripheral and coronary function using laser Doppler imaging and transthoracic echocardiography. Clin Sci. 2008;115:295-300. 